\title{
Catálisis en Baja California
}

\author{
J. Noé Díaz de León, * Luz A. Zavala-Sánchez*
}

RESUMEN: Este artículo tiene como objetivo mostrar la investigación en materia de catálisis llevada a cabo en Baja California, México. Se describen los centros de investigación y universidades que aportan conocimiento por medio de proyectos de ciencia básica, así como de innovación tecnológica en dicho tema. Se mencionan las diferentes líneas de investigación en catálisis desarrolladas por los investigadores de la región. Además, se destaca la investigación llevada a cabo en Ensenada, como ciudad del conocimiento, por contar con numerosas instituciones dedicadas a la ciencia.

PALABRAS CLAVE: Catálisis, Baja California, CNyn, UABC, CICESE.

ABSTRACT: This paper aims to show the catalysis research carried out in Baja California, Mexico. It describes the research centers and universities that contribute knowledge through basic science projects, as well as technological innovation in this area. The different lines of research in catalysis that are developed by the researchers of the region are mentioned. Also, it highlights the research carried out in Ensenada, as a city of knowledge, because its many institutions dedicated to science.

KEYWORDS: Catalysis, Baja California, CNyn, UABC, CICESE.

\section{Introducción}

Los catalizadores se definen como compuestos que reaccionan químicamente pero que no son consumidos en el transcurso de la reacción. ${ }^{1}$ Un material catalítico es aquel que ayuda a la reacción a llevarse a cabo de manera más eficiente disminuyendo la energía de activación de esa reacción. Los catalizadores heterogéneos se componen normalmente de una fase activa y un soporte que normalmente proporciona una alta superficie sobre la que se dispersa la fase activa, frecuentemente de tamaño nanométrico y, por lo tanto, la catálisis por definición es un fenómeno "nano". En muchos casos existen otros compuestos de superficie conocidos como promotores, que mejoran las características del catalizador optimizando su dispersión (promotores estructurales), dirigiendo la selectividad e incrementando de manera considerable

Recibido: 13 de febrero de 2016. Aceptado: 31 de marzo de 2016.

* Universidad Nacional Autónoma de México, Centro de Nanociencias y Nanotecnología, Km. 107 carretera Tijuana-Ensenada, C.P. 22800, Ensenada, Baja California, México. Correspondencia: J. N. Díaz De León (noejd@cnyn.unam.mx), Luz A. Zavala-Sánchez (luz_ zavala@outlook.com);

1 Tomás Cordero Alcántara y José Rodríguez Mirasol (Universidad de Málaga). Catalizadores para la protección del medio ambiente Rodríguez Jiménez, Juan José (dir.), Hacia un uso sostenible de los recursos naturales. Sevilla: Universidad Internacional de Andalucía, 2008. ISBN: 978-84-7993-048-6. 
Mundo Nano | CATÁLISIS EN MÉXICo | www.mundonano.unam.mx

10(18), enero-junio 2017 | DOI: 10.22201/ceiich.24485691e.2017.18.60055

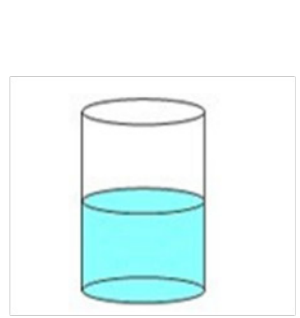

Homogénea

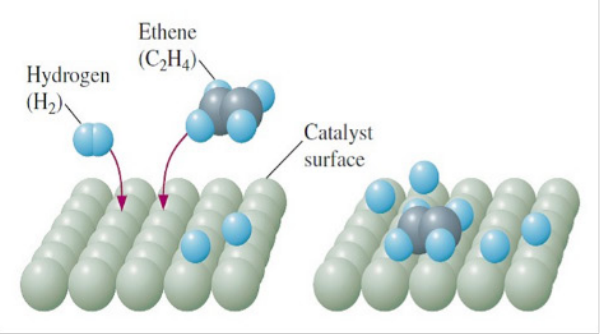

Heterogénea

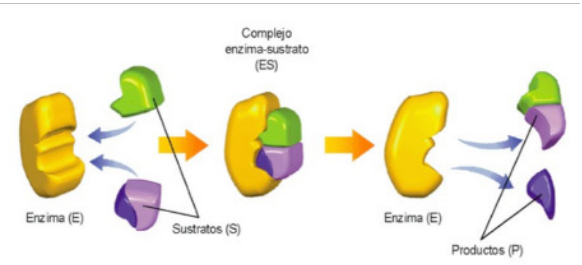

$E+S \rightarrow E S \rightarrow E+P$

Enzimática

FIGURA 1. Clasificación de las reacciones catalíticas.

la actividad catalítica (promotores químicos). La catálisis puede llevarse a cabo de múltiples formas, por ejemplo: a) la catálisis homogénea, es decir, cuando los reactivos y el catalizador se encuentran en la misma fase. En este tipo de catálisis generalmente los reactivos están en un medio acuoso y los catalizadores son frecuentemente pares ácido/base; b) la catálisis heterogénea en la cual los catalizadores son sólidos usualmente porosos y los reactivos pueden estar en fase gaseosa o líquida, y, c) se encuentra la catálisis de tipo "bio" en la cual se necesita de una enzima y un sustrato afín.

La importancia de la catálisis en el mundo es el desarrollo de nuevas soluciones tecnológicas que contribuyan a la mejora de la calidad de vida humana y potencien la competitividad de la industria química, dentro del concepto de química sostenible. En los últimos años, el espectro de la investigación y el desarrollo de catalizadores avanzados, tanto inorgánicos como enzimáticos, han dado como resultado procesos químicos innovadores. Ya sea para mejorar los sistemas de producción existentes, reducir la producción de especies contaminantes o evitar la contaminación de los efluentes que se emiten. Las aplicaciones principales son en materia de energía, protección medioambiental y síntesis selectiva de compuestos químicos.

\section{Baja California, punto de investigación científica e innovación tecnológica}

Baja California, estado ubicado en el extremo noroeste de México, se distingue de otros estados por un modelo económico y social fructífero y punto estratégico para el intercambio de bienes, recursos humanos y conocimiento con el país vecino, Estados Unidos. La variedad étnica y su consecuente diversidad cultural son los sellos distintivos de esta zona fronteriza, donde se encuentran tres ciudades principales Ensenada, Mexicali y Tijuana. Mexicali es la capital del estado mientras que Tijuana es la ciudad más grande de la entidad; esta singular característica ha dado origen a profundos fenómenos sociales cuyos efectos y manifestaciones son continuamente estudiados en 
prestigiadas instituciones mexicanas y estadounidenses. Más que un estado que marque diferencias es un punto de unión entre culturas.

\section{Entidades en las que se realiza investigación en Baja California}

Ensenada es actualmente la ciudad con más científicos por habitante de Méxi$\mathrm{Co}^{2}$ y ha sido declarada recientemente como Ciudad del Conocimiento. ${ }^{3}$ En Baja California existe una gran variedad de instituciones educativas con una muy diversa oferta. Por ejemplo, a nivel posgrado, hay dos centros del Consejo Nacional de Ciencia y Tecnología (CONACyT): el Centro de Investigación Científica y de Educación Superior de Ensenada (CICESE) y el Colegio de la Frontera Norte (COLEF), de entre ellos el primero ha sido catalogado como el centro CONACyT más grande de México. Tijuana cuenta con el Centro de Graduados e Investigación en Química del Instituto Tecnológico de Tijuana (CGIQ-ITT) y el Centro de Investigación en Tecnología Digital (CITEDI), el cual es una unidad del Instituto Politécnico Nacional (IPN). En Ensenada, se ubican el Centro de Nanociencias y Nanotecnología ( $\mathrm{CNyN}$ ) y el Instituto de Astronomía (IAUNAM), ambos dependientes de la Universidad Nacional Autónoma de México. Además de la Universidad Autónoma de Baja California (UABC).

FIGURA 2. Ubicación de las principales ciudades de Baja California y su escudo.
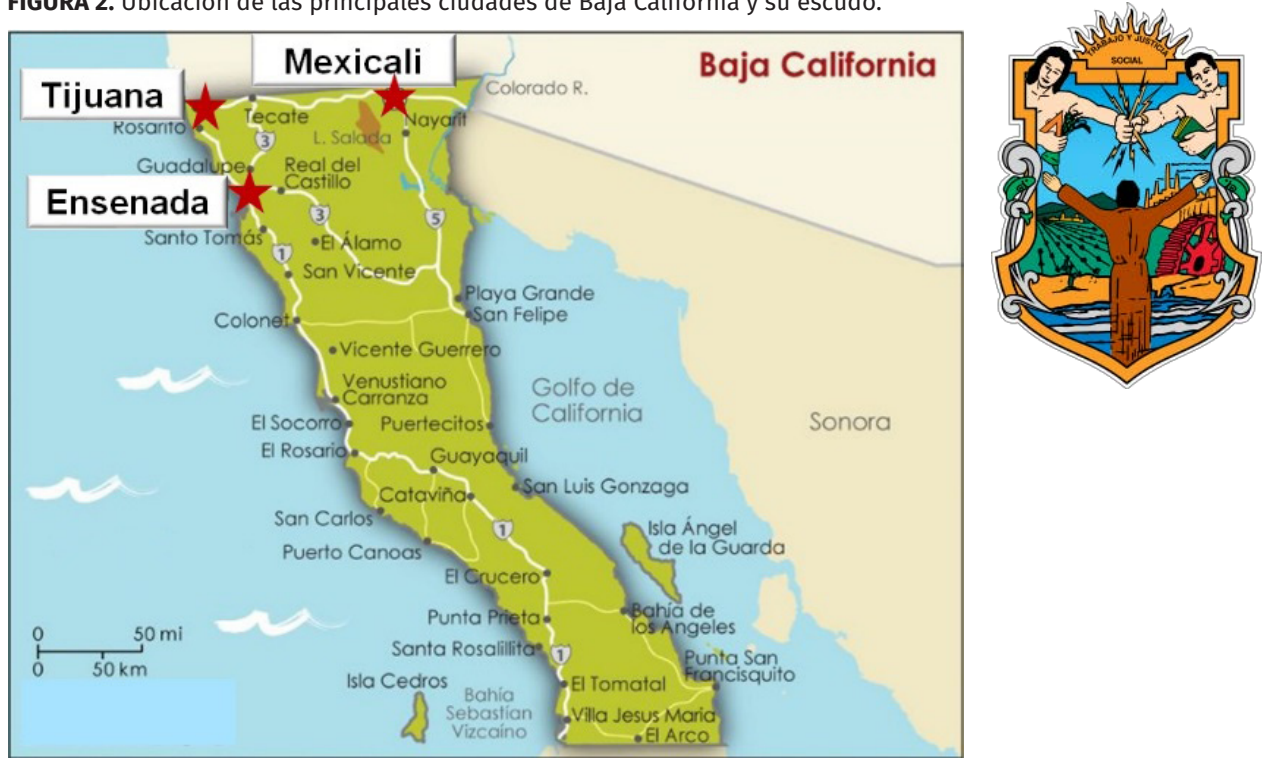

2 «Biotecnología - Invest in Baja». 〈www.investinbaja.gob.mx〉.

3 «Ensenada, Ciudad del Conocimiento - El Vigía». 
Mundo Nano | CATÁLISIS EN MÉxICo | www.mundonano.unam.mx

10(18), enero-junio 2017 | DOI: 10.22201/ceiich.24485691e.2017.18.60055

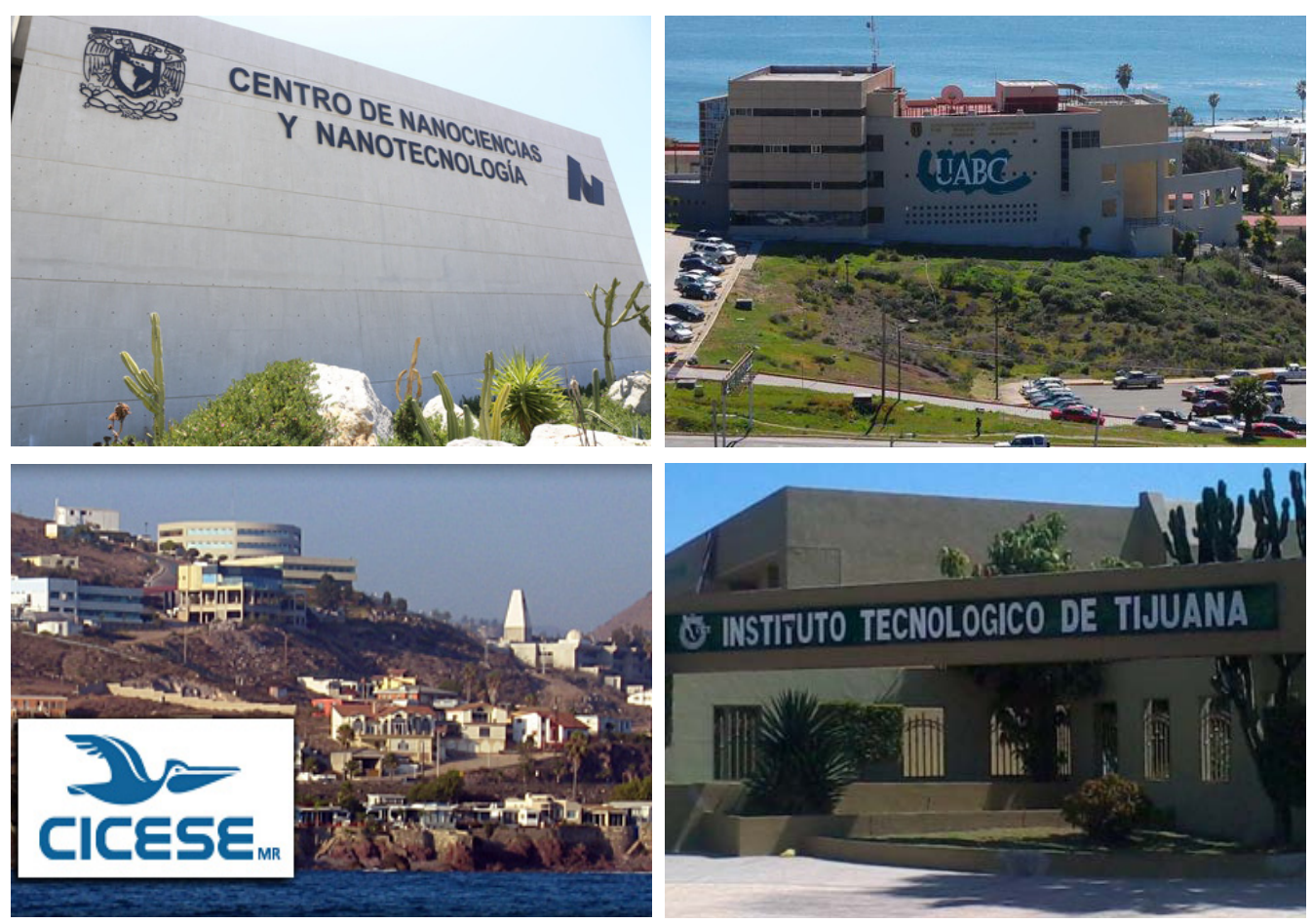

FIGURA 3. Instituciones que tienen programas de posgrado en Baja California.

\section{Oferta educativa}

Los programas de licenciatura y posgrado constituyen un esfuerzo por ampliar la oferta educativa con el propósito de atender las necesidades y participar en la búsqueda y generación de conocimiento nuevo no sólo para el país, sino para la comunidad internacional.

En Baja California se cuenta con siete programas académicos para licenciatura, maestría y doctorado. Cinco de ellos impartidos en la ciudad de Ensenada, en el CNyN, UABC Campus Ensenada y CICESE.

- Licenciatura en nanotecnología (CNyN, UNAM).

- Ingeniería en nanotecnología (UABC).

- Posgrado en ciencia e ingeniería de materiales (CNyN, UNAM).

- Posgrado en ciencias físicas (CNyN, UNAM).

- Posgrado en nanociencias (CICESE-CNyN-UnAM).

- Maestría y doctorado en ciencias e ingeniería (UABC Campus Ensenada, Tijuana y Mexicali).

- Maestría y doctorado en ciencias en químicas (CGIQ-ITT). 
Debido a esta gran diversidad de instituciones también se cuenta en Baja California con el Consejo Estatal de Ciencia y Tecnología (сосут) que promueve programas de desarrollo interinstitucionales procurando la vinculación y el compromiso de pensamiento y colaboración entre industrias, universidades y gobierno. Este organismo estableció el Premio Estatal de Ciencia y Tecnología que se otorga de manera anual como un reconocimiento público al trabajo realizado de manera individual o colectiva por científicos o tecnólogos bajacalifornianos o residentes en la Entidad.

Entre las principales aportaciones de los grupos de investigación existentes en la región de Baja California, se cuenta con el desarrollo de la preparación de nanomateriales y nanoestructuras utilizando técnicas sofisticadas de síntesis. Esta disciplina tiene un gran espectro de aplicaciones energéticas y ambientales, como son el desarrollo de catalizadores para motores de autos y nanotubos para almacenamiento de hidrógeno. En el ámbito de la catálisis existen muy variadas líneas de investigación en materiales, por ejemplo, para fotocatálisis, electroquímica, hidrotratamiento, producción de hidrogeno y energías limpias, química fina, reformación, deshidratación de alcoholes, biocatálisis, así como el estudio de catálisis teórica y modelamiento (figura 4).

\section{Centro de Nanociencias y Nanotecnología de la UNAM}

El Cnyn (antes Centro de Ciencias de la Materia Condensada) de la UNAM ha enfocado su esfuerzo en el estudio de la materia a nivel atómico, evolucionando hacia la modificación de la misma logrando crear una gran diversidad de nanoestructuras con diferentes morfologías aplicadas a catalizadores nanoestructurados de aleaciones metálicas. Las actividades en el CNyN se lle-

FIGURA 4. Líneas de investigación en materia de catálisis en Baja California.

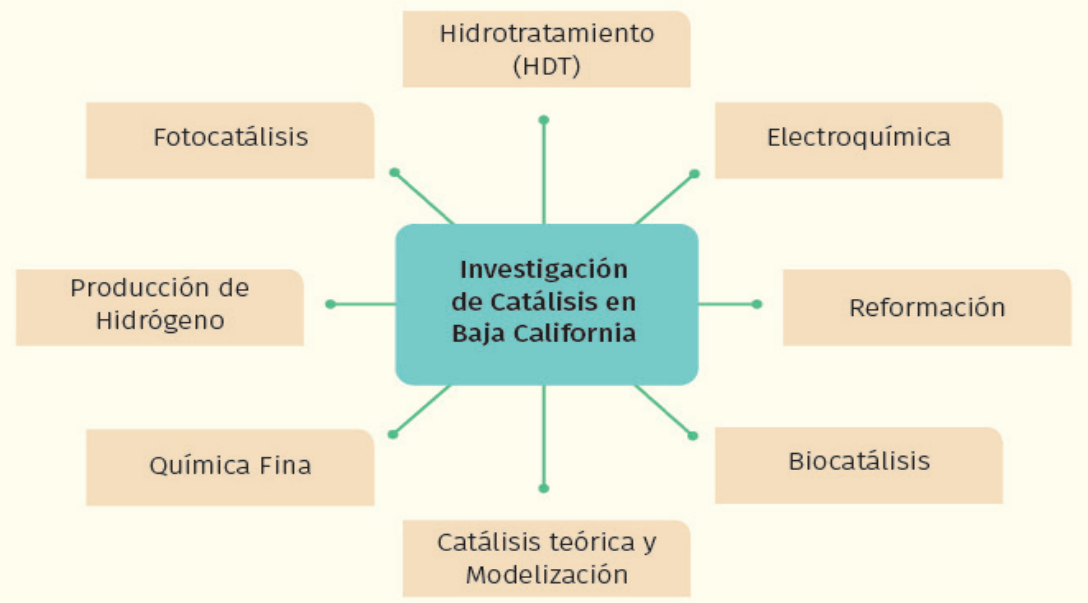


van a cabo en diferentes departamentos, como son los de Bionanotecnología, Física, Fisicoquímica de Nanomateriales, Materiales Avanzados, Nanocatálisis, y Nanoestructuras, y los laboratorios especializados de apoyo a la investigación. Actualmente, el Departamento de Nanocatálisis cuenta con nueve académicos y dieciocho alumnos de licenciatura y posgrado; está compuesto por los doctores Amelia Olivas Sarabia, Felipe Castillón Barraza, Trino Armando Zepeda Partida, Vitalli Petranovskii, Sergio Fuentes Moyado, Andrey Simakov, Erick Flores Aquino, Takeshi Ogawa Murata y Jorge Noé Díaz de León Hernández. Cada uno con líneas de investigación bien definidas que se detallan en la tabla 1.

Tabla 1. Miembros del Departamento de Nanocatálisis del cnyn y sus líneas de investigación.

\begin{tabular}{|c|c|}
\hline INVESTIGADOR & LÍNEA DE INVESTIGACIÓN \\
\hline $\begin{array}{l}\text { Amelia Olivas } \\
\text { Sarabia }\end{array}$ & $\begin{array}{c}\text { Sintesis de materiales catalíticos para reacciones de CO y su } \\
\text { caracterización fisicoquímica. }\end{array}$ \\
\hline $\begin{array}{c}\text { Felipe Castillón } \\
\text { Barraza }\end{array}$ & $\begin{array}{c}\text { Síntesis de catalizadores de tres vías de Pd (Pd-TWC). } \\
\text { Catalizadores para transformación de CO, catalizadores } \\
\text { enzimáticos y deshidrogenación oxidativa de compuestos } \\
\text { orgánicos. }\end{array}$ \\
\hline $\begin{array}{l}\text { Trino A. Zepeda } \\
\text { Partida }\end{array}$ & $\begin{array}{l}\text { Generación de energías limpias por medio del estudio de } \\
\text { catalizadores para las reacciones de desplazamiento de vapor } \\
\text { de agua (WGS), para la obtención de hidrógeno y proceso Fischer- } \\
\text { Tropsch a partir de gases de síntesis }\left(\mathrm{CO} \text { y } \mathrm{H}_{2}\right) \text {, así como para la } \\
\text { remoción de azufre de cortes petroleros. }\end{array}$ \\
\hline $\begin{array}{c}\text { Vitalli } \\
\text { Petranovskii } \\
\end{array}$ & $\begin{array}{l}\text { Síntesis y estudio de las propiedades físicas-químicas y } \\
\text { estructurales de cúmulos de metales en las zeolitas. }\end{array}$ \\
\hline $\begin{array}{l}\text { Sergio Fuentes } \\
\text { Moyado }\end{array}$ & $\begin{array}{c}\text { Química, cinética y catálisis en ingeniería química, refinación del } \\
\text { petróleo, petroquímica, fisicoquímica de superficies y } \\
\text { nanotecnología. Su línea más importante es el estudio de } \\
\text { materiales en estado sulfuro para diversas aplicaciones. }\end{array}$ \\
\hline Andrey Simakov & $\begin{array}{l}\text { Desarrollo de nuevos catalizadores heterogéneos para la } \\
\text { protección del medio ambiente, diseño de procesos novedosos en } \\
\text { biocatálisis y biotecnología, investigación de la cinética y de los } \\
\text { mecanismos cinéticos de catálisis heterogénea con aplicación de } \\
\text { técnicas experimentales avanzadas como in situ y operando. }\end{array}$ \\
\hline $\begin{array}{l}\text { Takeshi Ogawa } \\
\text { Murata }\end{array}$ & $\begin{array}{l}\text { Síntesis y modelado molecular de los catalizadores basados en } \\
\text { complejos de polímeros hiperramificados con metales de } \\
\text { transición. El estudio del efecto dendrimérico sobre diferentes } \\
\text { catalizadores. }\end{array}$ \\
\hline $\begin{array}{l}\text { Jorge Noé Díaz } \\
\text { de León } \\
\text { Hernández }\end{array}$ & $\begin{array}{c}\text { Preparación, caracterización y evaluación catalítica de soportes y } \\
\text { catalizadores para la producción de diésel de ultra-bajo azufre. } \\
\text { Desarrollo de nanomateriales 1D de óxidos de metales típicos. } \\
\text { Desarrollo de infraestructura para la evaluación catalítica de } \\
\text { materiales de hidrotratamiento a escala micro y piloto bajo } \\
\text { condiciones industriales, así como materiales para la } \\
\text { deshidratación catalítica de alcoholes. }\end{array}$ \\
\hline
\end{tabular}


A su vez, el Dr. Sergio Fuentes preside el grupo de investigación en sulfuros al que están asociados los doctores Trino A. Zepeda, Vitalli Petranovskii, Jorge Noé Díaz de León y Gabriel Alonso-Núñez del Departamento de Nanoestructuras. Los académicos invitados Dra. Elena Smolentzeva y Dra. Yulia Kotolevich, los doctores Brenda Acosta, Joel Antúnez, Miguel Estrada y Joel Rojas quienes están realizando sus estancias posdoctorales. Cabe mencionar que el doctor Sergio Fuentes (figura 5) obtuvo en 2016 el Premio de Ciencia y Tecnología del estado de Baja California, el Premio a la Trayectoria Científica de la Federación Iberoamericana de Sociedades de Catálisis (FISOCAT) y fue nombrado secretario de la International Association of Catalysis Societies (IACS).

Además de los integrantes del Departamento de Nanoca-

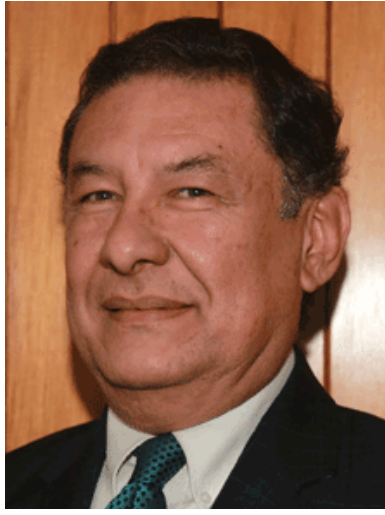

FIGURA 5. Profesor Sergio Fuentes Moyado. tálisis que se dedican enteramente al tópico de este trabajo, los doctores Rafael Vásquez Duhalt, Nina Bogdanchikova, Karla O. Juárez Moreno, Homero Galván Martínez, Sergio Águila Puentes y Gabriel Alonso Núñez pertenecientes a otros departamentos del CNYN, también realizan investigaciones relacionadas con catálisis. Algunas de sus líneas de investigación se detallan en la tabla 2.

Tabla 2. Miembros del CNyn en otros departamentos que desarrollan investigación en catálisis.

\begin{tabular}{|c|c|}
\hline INVESTIGADOR & LÍNEA DE INVESTIGACIÓN \\
\hline $\begin{array}{l}\text { Rafael Vázquez } \\
\text { Duhalt }\end{array}$ & $\begin{array}{c}\text { Biotecnología ambiental. Degradación biocatalítica de } \\
\text { contaminantes con motores autopropulsados de liberación } \\
\text { enzimática. }\end{array}$ \\
\hline $\begin{array}{c}\text { Nina } \\
\text { Bogdanchikova }\end{array}$ & $\begin{array}{c}\text { Oxidación de propeno y propano, oxidación de co, así como el } \\
\text { estudio de las propiedades fisicoquímicas de nano partículas y } \\
\text { cúmulos de plata y oro. }\end{array}$ \\
\hline $\begin{array}{l}\text { Karla O. Juárez } \\
\text { Moreno }\end{array}$ & Biocatálisis enzimática. \\
\hline $\begin{array}{l}\text { Homero Galván } \\
\text { Martínez }\end{array}$ & Modelado cuántico para catálisis. \\
\hline $\begin{array}{l}\text { Sergio A. Águila } \\
\text { Puentes }\end{array}$ & $\begin{array}{l}\text { Biocatálisis, la inmovilización de enzimas en soportes } \\
\text { nanoestructurados. Estudios biocatalíticos de enzimas } \\
\text { oxidorreductasas. Diseño de nanopartículas pseudovirales con } \\
\text { actividad biocatalítica. }\end{array}$ \\
\hline $\begin{array}{c}\text { Gabriel Alonso } \\
\text { Núñez }\end{array}$ & $\begin{array}{c}\text { Síntesis y caracterización de nanoestructuras de metales nobles y } \\
\text { nanotubos de carbón con aplicación en catálisis y electroquímica. } \\
\text { Síntesis y caracterización de sulfuros de metales de transición } \\
\left(\mathrm{MoS}_{2}, \mathrm{ReS}_{2}, \mathrm{RuS}_{2} \text { y WS } \mathrm{WS}_{2} \text { ) con aplicación en reacciones de HDS de }\right. \\
\text { compuestos azufrados, desarrollo de nuevos materiales } \\
\text { nanoestructurados como: nanotubos de carbono, óxidos ( } \mathrm{TiO}_{2} \text { y } \\
\left.\mathrm{SiO}_{2}\right) \text { y metales nobles (Pt, Rh, Pd, Au, Ru) con aplicación en celdas } \\
\text { de combustible de intercambio iónico. }\end{array}$ \\
\hline
\end{tabular}



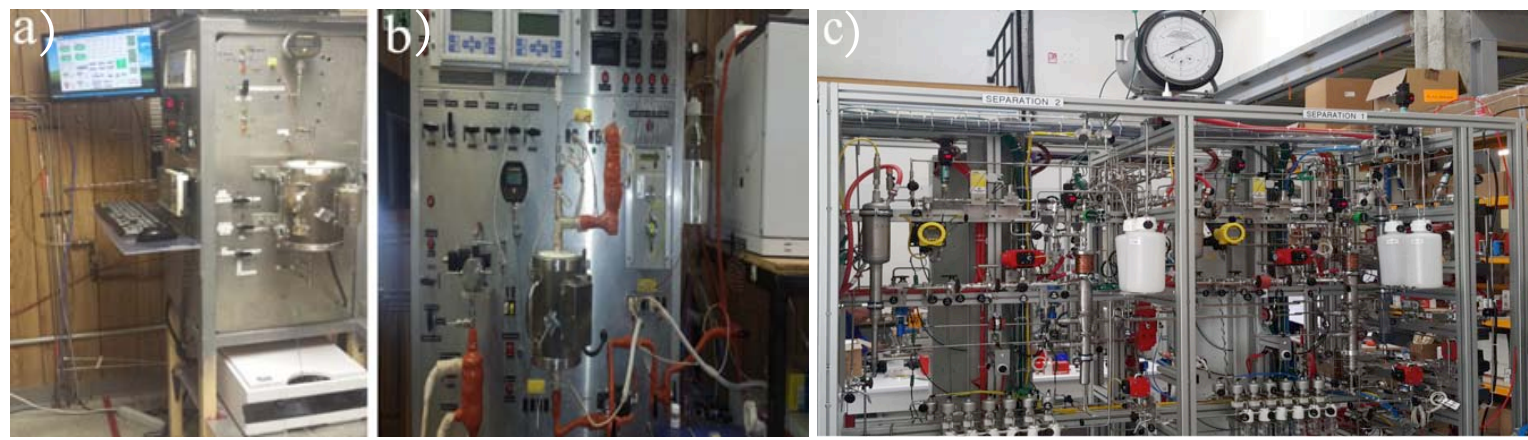

FIGURA 6. a) Reactor continuo de cama empacada para reacciones de hidrodesulfuración; b) reactor para reacciones de Fischer-Tropsh, reformación y desplazamiento de vapor de agua, y, c) planta piloto para evaluaciones a escala de banco tipo Vinci.

Se debe destacar que en conjunto los académicos del cNyn que mantienen líneas de investigación en catálisis han logrado publicar en 2016 más de 70 artículos en revistas indizadas de alto impacto. Con lo cual contribuyen al desarrollo de la catálisis en México de manera activa.

La infraestructura con la que cuenta el CNyN fue enumerada previamente en el trabajo de Barberena y colaboradores, ${ }^{4}$ pero además, para el desarrollo de las investigaciones en catálisis se cuentan con reactores especializados para pruebas cinéticas de materiales. Existen varios reactores por lotes tipo Parr, un reactor de flujo continuo para las reacciones de FischerTropsh, reformación y desplazamiento de vapor de agua, un reactor de flujo continuo descendente en fase gas para reacciones de deshidratación de alcoholes y desulfuración de moléculas modelo tipo gasolina a presión atmosférica. Un reactor trifásico de cama empacada para hidrodesulfuración de moléculas modelo tipo diésel y cargas reales. Un reactor para hidrogenaciones selectivas y un reactor para oxidación de $\mathrm{CO}$, entre otros. Cabe mencionar que con excepción de los sistemas Parr, todos los reactores fueron desarrollados por los académicos involucrados en esa línea específica de investigación. Finalmente, el centro cuenta con una planta piloto a nivel escala de banco de reciente adquisición con VINCI technologies por medio del proyecto SENER-CONACyT 117373 dirigido por el Dr. Sergio Fuentes. Misma que puede ser utilizada para evaluación de prototipos de catalizadores industriales en reacciones de remoción ultra-profunda de moléculas azufradas presentes en cargas reales tipo diésel, hidrogenación selectiva de compuestos aromáticos, hidrodenitrogenación, hidrodemetalización, hidrodeoxigenación, entre otras.

${ }^{4}$ Centro de Nanociencias y Nanotecnología-UnAM, Irene Barberena Rojas, Oscar Edel Contreras López y Leonardo Morales de la Garza, Mundo Nano. Revista Interdisciplinaria en Nanociencias y Nanotecnología, vol. 9, núm. 16, enero-junio, 2016, 30-48. 
La colaboración científica constituye un aspecto esencial en la actividad profesional de los investigadores del CNyN. Entre otros beneficios, la cooperación permite alcanzar de forma más rápida y eficiente un fin común; incluso puede ser en ocasiones la única forma para alcanzarlo. En los últimos años se ha intensificado hasta tal punto la especialización del conocimiento y los recursos necesarios para abordar los problemas de la investigación, que hacen de la misma un factor indispensable. ${ }^{5}$ De esta manera el grupo de Nanocatálisis mantiene una estrecha colaboración al interior del CNyN con diferentes departamentos como el de Bionanotecnología ${ }^{6}$ o física teórica ${ }^{7}$ (ver figura 7).

Asimismo, los investigadores del CNyN mantienen una amplia colaboración con instituciones locales y nacionales, así como otros centros e institutos de la UNAM. En el esquema de la figura 8 se muestran algunas de las colaboraciones que mantienen los investigadores que desarrollan catálisis en CNyN con instituciones nacionales.

FIGURA 7. Trabajos publicados por miembros del Departamento de Nanocatálisis en colaboración interna con otros departamentos del Cnyn. Izq.(véase nota 6), Der. (véase nota 7).

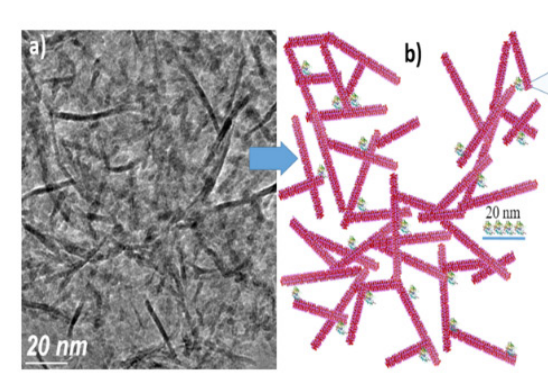

Bionanotecnología
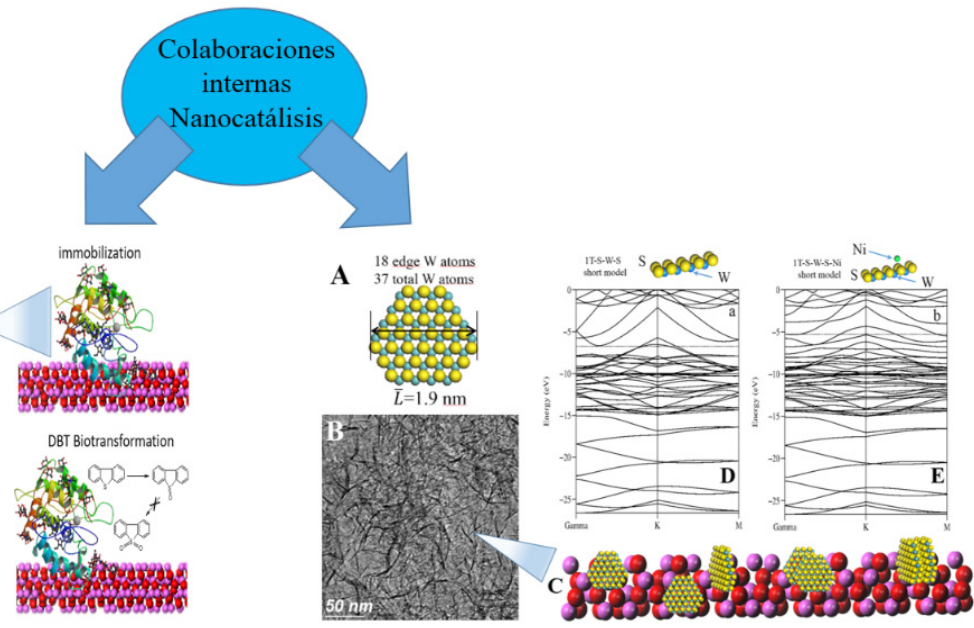

Física Teórica

${ }^{5}$ González Alcaide, G., Gómez Ferri, J. (2014). La colaboración científica: principales líneas de investigación y retos de futuro. Revista Española de Documentación Científica, 37 (4): e062 doi: http://dx.doi.org/10.3989/redc.2014.4.1186.

6 Karla Juarez-Moreno, J. Noé Díaz de León, Trino A. Zepeda, Rafael Vazquez-Duhalt, Sergio Fuentes. (2015). Oxidative transformation of dibenzothiophene by chloroperoxidase enzyme immobilized on (1D) $-\gamma-\mathrm{Al}_{2} \mathrm{O}_{3}$ nanorods. Journal of Molecular Catalysis B Enzymatic, 115, 90-95.

7 J. N. Díaz de León, T.A. Zepeda, G. Alonso-Núñez, D.H. Galván, B. Pawelec y S. Fuentes. (2015). Insight of $1 \mathrm{D} \gamma-\mathrm{Al}_{2} \mathrm{O}_{3}$ nanorods decoration by NiWS nano-slabs in ultra-deep hydrodesulfurization catalysts. Journal of Catalysis, 321, 51-61. 
Mundo Nano | CATÁLISIS EN MÉXICo | www.mundonano.unam.mx

10(18), enero-junio 2017 | DOI: 10.22201/ceiich.24485691e.2017.18.60055

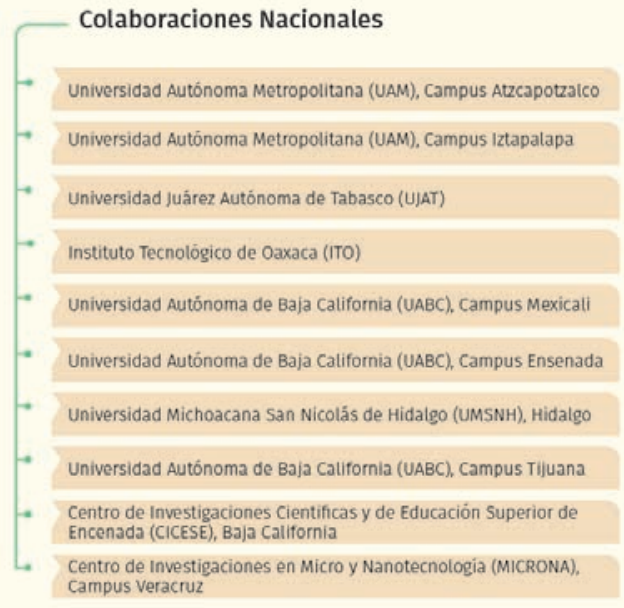

FIGURA 8. Colaboraciones del Departamento de Nanocatálisis con instituciones nacionales.

De la misma forma, las líneas de investigación sobre catálisis les han permitido a los investigadores mantener una intensa vinculación con instituciones de alto prestigio en el extranjero, con el objetivo no sólo de estancias académicas sino también de la transferencia de tecnología y conocimiento a nuestro país. Algunas de las instituciones con las que actualmente se tiene vinculación se enumeran en el esquema de la figura 9.

FIGURA 9. Colaboraciones del Departamento de Nanocatálisis con instituciones internacionales.

\section{Colaboraciones Internacionales}

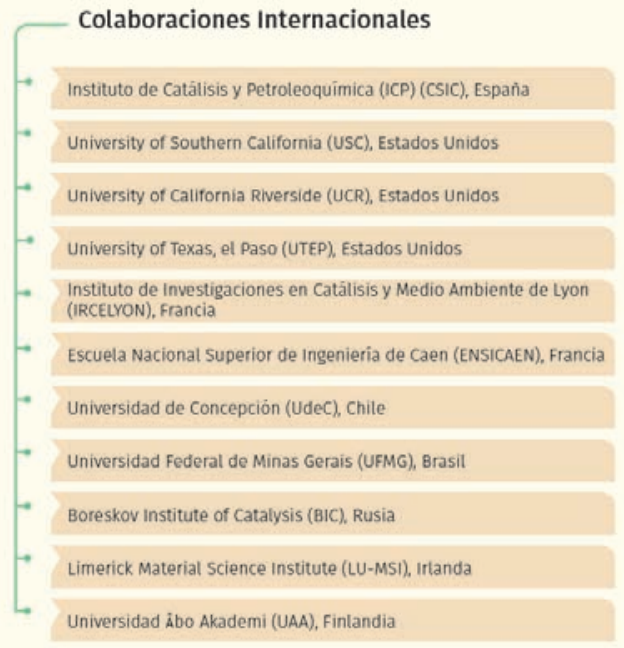


Uno de los ejemplos más recientes de transferencia de tecnología es la implementación de una cámara de vacío para transferencia de materiales en estado sulfuro para su análisis in-situ y caracterización por medio de espectroscopía fotoelectrónica de rayos X (XPS). Misma que fue realizada en colaboración con el Instituto de Investigación en Catálisis y Medio Ambiente de Lyon, Francia, en una estancia realizada por la M. en C. Luz Zavala-Sánchez con el Dr. Luis Cárdenas como parte de su participación en el proyecto SENER-CONACYT 117373.

\section{Universidad Autónoma de Baja California}

En el caso de la UABC los inicios de la investigación científica en cuanto a catálisis se remontan a la creación de la Facultad de Ciencias Químicas e Ingeniería UABC-Tijuana, que fue fundada el 18 de febrero de 1974 y que durante nueve años ofreció sólo el programa de licenciatura en química. En el año de 1983 se aumentó la oferta educativa, ofreciendo ahora la carrera de químico industrial y la de ingeniero químico. En 2002, la oferta educativa nuevamente crece, estableciéndose el Programa de Ingeniero Industrial. Un año después se lanza el Programa de Maestría y Doctorado en Ciencias e Ingeniería, ofrecido como plan institucional tanto en la Facultad de Ingeniería en el campus Ensenada como en Mexicali. Desde sus inicios el cuerpo de posgrado en química de materiales tiene como eje el estudio de la síntesis, caracterización y medición de la actividad catalítica de catalizadores sin soporte en las reacciones modelo de hidrotratamiento, particularmente hidrogenación del ciclohexeno y la hidrodesulfuración del dibenzotiofeno. Los académicos asociados a esta línea de investigación son los doctores Juan Cruz Reyes quien funge como líder del cuerpo académico, el Dr. José Mario del Valle Granados (figura 10) y el Dr. Raúl Romero Rivera.

FIGURA 10. De izquierda a derecha los doctores Gabriel Alonso-Núñez (CNyn-UnAM), Raúl Romero (UABC), Juan Cruz-Reyes (UABC), Hugo Tiznado (CNyN-UNAM) y Mario del Valle (UABC).

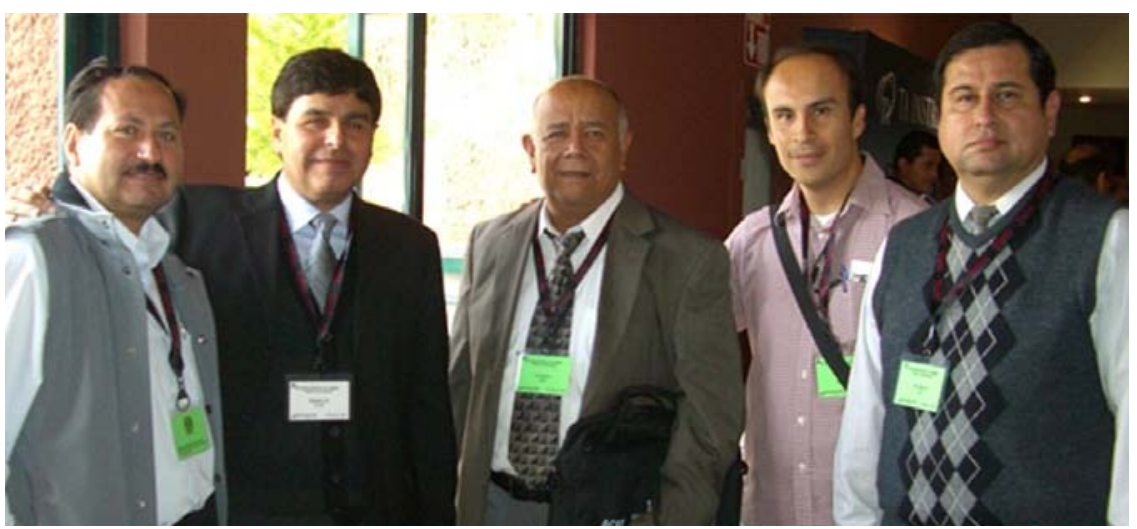


En el ámbito de las colaboraciones nacionales, los integrantes del cuerpo académico mantienen relaciones académicas con investigadores de otros centros de investigación nacionales, como son:

- Centro de Nanociencias y Nanotecnología (CNYN, UnAM, Ensenada, B.C.).

- Centro de Investigación en Materiales Avanzados (cimav, Chihuahua).

- Instituto Potosino de Investigación Científica y Tecnológica (IPCyT, San Luis Potosí).

- Destacando los siguientes académicos como colaboradores nacionales.

- Dr. Gabriel Alonso Núñez (CNyn, UnAM, Ensenada).

- Dr. Sergio Fuentes (CNyn, UnAM, Ensenada).

- Dr. Miguel Avalos Borja (IPCyT, S.L.P.).

- Dr. Francisco Paraguay Delgado (cimav, Chihuahua).

- Dr. Hugo Tiznado (cNyN, UnAM, Ensenada).

Del mismo modo, en el campus Ensenada se lleva a cabo catálisis por parte de la Dra. Eunice Vargas Viveros. Algunas de las líneas de investigación desarrolladas por los investigadores mencionados se detallan en la tabla 3.

\section{Centro de Graduados e Investigación en Química del Instituto Tecnológico de Tijuana}

En el Centro de Graduados se encuentran los doctores Edgar Reynoso Soto, Yadira Gochi, Mercedes Teresita Oropeza Guzmán y Rosa María Félix Navarro. Los temas de investigación que desarrollan en cuanto a catálisis son muy variados, pero principalmente se dedican al desarrollo de materiales para fo-

Tabla 3. Miembros de la UABC que desarrollan investigación en catálisis.

\begin{tabular}{|c|c|}
\hline INVESTIGADOR & LÍNEA DE INVESTIGACIÓN \\
\hline Juan Cruz Reyes & $\begin{array}{c}\text { Síntesis y caracterización de sulfuros bimetálicos con estructura } \\
\text { tipo grafeno de interés catalítico. Síntesis de catalizadores sin } \\
\text { soporte en las reacciones modelo de HDT, particularmente: } \\
\text { hidrogenación del ciclohexeno e hidrodesulfuración del } \\
\text { dibenzotiofeno. }\end{array}$ \\
\hline $\begin{array}{c}\text { Mario del Valle } \\
\text { Granados }\end{array}$ & $\begin{array}{c}\text { Síntesis de catalizadores sin soporte en las reacciones modelo de } \\
\text { HDT. Hidrodesulfuración de compuestos azufrados con sulfuros y } \\
\text { catalizadores derivados de tiometalatos de aminas cuaternarias. }\end{array}$ \\
\hline $\begin{array}{c}\text { Eunice Vargas } \\
\text { Viveros }\end{array}$ & $\begin{array}{c}\text { Síntesis y aplicación de nanopartículas de oro dispersadas en } \\
\text { ónidos metálicos para reacciones de oxidación de CO, reacción de } \\
\text { desplazamiento de vapor de agua (WGS) y oxidación selectiva de } \\
\text { compuestos orgánicos. }\end{array}$ \\
\hline
\end{tabular}


tocatálisis, electrocatálisis y remediación de efluentes. Algunas de sus líneas de investigación se detallan en la tabla 4.

Por último, los autores quisieran expresar su agradecimiento a los académicos que colaboraron con este trabajo, mencionando de manera escrita o verbal sus áreas de interés, colaboraciones y sus líneas de investigación. Asimismo, queremos invitar a los académicos que hacen investigación en catálisis radicados en el estado de Baja California y que no fueron mencionados en esta recopilación, a que se comuniquen con nosotros para considerarlos en futuros trabajos, así como para invitarlos a formar parte de la Académica de Catálisis México (ACAT).

Tabla 4. Miembros del Centro de Graduados e Investigación en Química que desarrollan investigación en catálisis.

\begin{tabular}{|c|c|}
\hline INVESTIGADOR & LÍNEA DE INVESTIGACIÓN \\
\hline $\begin{array}{l}\text { Edgar Reynoso } \\
\text { Soto }\end{array}$ & $\begin{array}{l}\text { Síntesis, caracterización y aplicación de nanomateriales. Los } \\
\text { proyectos en los que está desarrollando su investigación se } \\
\text { enfocan a la síntesis de nanoestructuras de carbono, } \\
\text { nanopartículas metálicas y bimetálicas, así como la síntesis de } \\
\text { dióxido de titanio dopado con metales de transición. Desarrollo } \\
\text { de celdas de combustible de hidrógeno y metanol, celdas solares } \\
\text { sensibilizadas con colorantes, electrocatalizadores para la } \\
\text { destrucción de contaminantes orgánicos, fotocatálisis } \\
\text { heterogénea para destrucción de colorantes en medios acuosos. }\end{array}$ \\
\hline Yadira Gochi & $\begin{array}{c}\text { Desarrollo de nuevos materiales y dispositivos de energía, } \\
\text { particularmente con base en la síntesis y caracterización de } \\
\text { nanoestructuras de carbono (nanotubos y grafeno) y } \\
\text { nanopartículas metálicas (elementos de transición) como } \\
\text { catalizadores de reacciones químicas específicas y } \\
\text { electrocatalizadores de celdas de combustible de membrana de } \\
\text { electrolito polimérico, así como el estudio de materiales } \\
\text { compuestos. }\end{array}$ \\
\hline $\begin{array}{c}\text { Mercedes } \\
\text { Teresita Oropeza } \\
\text { Guzmán }\end{array}$ & $\begin{array}{c}\text { Estudio de procesos electrocinéticos aplicados a la } \\
\text { descontaminación de suelos con metales e hidrocarburos. } \\
\text { Innovaciones en la preparación de electrocatalizadores DSA para } \\
\text { generación de radicales OH y oxígeno, como vía de mejora de } \\
\text { celdas de electroflotación, electrocoagulación electroxidación y } \\
\text { fotoelectroxidación. }\end{array}$ \\
\hline $\begin{array}{c}\text { Rosa María Félix } \\
\text { Navarro }\end{array}$ & $\begin{array}{c}\text { Síntesis de nanomateriales para aplicaciones en la detección y } \\
\text { remoción de contaminantes del medio ambiente, detección de } \\
\text { moléculas de interés biológico, electrocatalizadores para celdas } \\
\text { de combustible y celdas solares. Diseño de reactores } \\
\text { electroquímicos para la síntesis electroquímica de agentes } \\
\text { químicos para el tratamiento de aguas. } \\
\text { Diseño de reactores electroquímicos para la remoción de metales } \\
\text { pesados tóxicos de soluciones acuosas. Procesos de oxidación } \\
\text { avanzada para el tratamiento de aguas. }\end{array}$ \\
\hline
\end{tabular}




\section{Sitios de interés}

- Centro de Investigación Científica y de Educación Superior de Ensenada $\langle$ http://www.cicese.edu.mx $>$

- Centro de Graduados e Investigación en Química del Instituto Tecnológico de Tijuana $\langle$ http://cgiq.tectijuana.mx $>$

- Centro de Nanociencias y Nanotecnología $\langle$ https://www.cnyn.unam.mx >

- Universidad Autónoma de Baja California 〈http://www.uabc.mx>

- Consejo Estatal de Ciencia y Tecnología de Baja California <https://sites.google.com/a/uabc.edu.mx/cisalud/convocatoria/ consejo-de-ciencia-y-tecnologia-baja-california $>$

- Academia de Catálisis de México $\langle$ http://www.acat.org.mx >

\section{Páginas de investigadores}

\section{Centro de Nanociencias y Nanotecnología de la UNAM}

- Dra. Amelia Oliva Sarabia <https://www.cnyn.unam.mx/index.php?option=com_perfil\&view $=$ perfil\&Itemid=56\&uid $=108 \&$ lang $=$ en $>$

- Dr.Felipe Castillón Barraza <https://www.cnyn.unam.mx/index.php?option=com_perfil\&view =perfil\&Itemid=56\&uid=70\&lang=en>

- Dr. Trino Armando Zepeda Partida <https://www.cnyn.unam.mx/index.php?option=com_perfil\&view =perfil\&Itemid=56\&uid=138\&lang=en $>$

- Dr. Vitalli Petranovskii <https://www.cnyn.unam.mx/index.php?option=com_perfil\&view =perfil\&Itemid=56\&uid=115\&lang=en > 
- Dr. Sergio Fuentes Moyado

<https://www.cnyn.unam.mx/index.php?option=com_perfil\&view =perfil\&Itemid=56\&uid=84\&lang=en $>$

- Dr. Andrey Simakov <https://www.cnyn.unam.mx/index.php?option=com_perfil\&view =perfil\&Itemid=56\&uid=125\&lang=en $>$

- Dr. Jorge Noé Díaz de León Hernández <https://www.cnyn.unam.mx/index.php?option=com_perfil\&view =perfil\&Itemid=56\&uid=143\&lang=en $>$

- Dr. Rafael Vásquez Duhault <https://www.cnyn.unam.mx/index.php?option=com_perfil\&view =perfil\&Itemid=56\&uid=167\&lang=en $>$

- Dra. Nina Bogdanchikova <https://www.cnyn.unam.mx/index.php?option=com_perfil\&view =perfil\&Itemid=56\&uid=65\&lang=en $>$

- Dra. Karla O. Juárez Moreno <https://www.cnyn.unam.mx/index.php?option=com_perfil\&view =perfil\&Itemid=56\&uid=161\&lang=en $>$

- Dr. Homero Galván Martínez <https://www.cnyn.unam.mx/index.php?option=com_perfil\&view =perfil\&Itemid=56\&uid=85\&lang=en $>$

- Dr. Sergio Águila Puentes <https://www.cnyn.unam.mx/index.php?option=com_perfil\&view $=$ perfil\&Itemid $=56 \&$ uid $=163 \&$ lang $=$ en $>$

- $\quad$ Dr. Gabriel Alonso Núñez <https://www.cnyn.unam.mx/index.php?option=com_perfil\&view =perfil\&Itemid=56\&uid=63\&lang=en $>$

\section{Centro de Graduados e Investigación Química del Instituto}

Tecnológico de Tijuana

- Dr. Edgar Reynoso Soto <http://cgiqtectijuana.mx/?page_id=1231>

- Dra. Yadira Gochi <http://cgiqtectijuana.mx/?page_id=1235> 
Mundo Nano | CATÁLISIS EN MÉXICo | www.mundonano.unam.mx

10(18), enero-junio 2017 | DOI: 10.22201/ceiich.24485691e.2017.18.60055

- Dra. Mercedes Teresita Oropeza Guzmán

$\langle$ http://cgiqtectijuana.mx/?page_id=735>

- Dra. Rosa María Félix Navarro

<http://cgiqtectijuana.mx/?page_id=739> 